University of Texas at El Paso

ScholarWorks@UTEP

$6-2001$

\title{
Towards More Realistic (e.g., Non-Associative) And- and Or- Operations in Fuzzy Logic
}

Jesus Martinez

Leopoldo Macias

Ammar Esper

Jesus Chaparro

Vick Alvarado

See next page for additional authors

Follow this and additional works at: https://scholarworks.utep.edu/cs_techrep

Part of the Computer Engineering Commons

Comments:

UTEP-CS-01-18.

Published in Proceedings of the 2001 IEEE Systems, Man, and Cybernetics Conference, Tucson, Arizona, October 7-10, 2001, pp. 2187-2192.

\section{Recommended Citation}

Martinez, Jesus; Macias, Leopoldo; Esper, Ammar; Chaparro, Jesus; Alvarado, Vick; Starks, Scott A.; and Kreinovich, Vladik, "Towards More Realistic (e.g., Non-Associative) And- and Or-Operations in Fuzzy Logic" (2001). Departmental Technical Reports (CS). 396.

https://scholarworks.utep.edu/cs_techrep/396

This Article is brought to you for free and open access by the Computer Science at ScholarWorks@UTEP. It has been accepted for inclusion in Departmental Technical Reports (CS) by an authorized administrator of ScholarWorks@UTEP.For more information, please contact Iweber@utep.edu. 


\section{Authors}

Jesus Martinez, Leopoldo Macias, Ammar Esper, Jesus Chaparro, Vick Alvarado, Scott A. Starks, and Vladik Kreinovich 


\title{
TOWARDS MORE REALISTIC (e.g., NON-ASSOCIATIVE) AND- AND OR-OPERATIONS IN FUZZY LOGIC
}

\author{
JESUS MARTINEZ, LEOPOLDO MACIAS, AMMAR ESPER \\ JESUS CHAPARRO, VICK ALVARADO, SCOTT A. STARKS, and VLADIK KREINOVICH
}

\author{
NASA Pan-American Center for Earth and Environmental Studies \\ University of Texas, El Paso, TX 79968, USA, vladik@cs.utep.edu
}

\begin{abstract}
How is fuzzy logic usually formalized? There are many seemingly reasonable requirements that a logic should satisfy: e.g., since $A \& B$ and $B \& A$ are the same, the corresponding and-operation should be commutative. Similarly, since $A \& A$ means the same as $A$, we should expect that the and-operation should also satisfy this property, etc. It turns out to be impossible to satisfy all these seemingly natural requirements, so usually, some requirements are picked as absolutely true (like commutativity or associativity), and others are ignored if they contradict to the picked ones.

This idea leads to a neat mathematical theory, but the analysis of real-life expert reasoning shows that all the requirements are only approximately satisfied. we should require all of these requirements to be satisfied to some extent. In this paper, we show the preliminary results of analyzing such operations. In particular, we show that non-associative operations explain the empirical $7 \pm 2$ law in psychology according to which a person can normally distinguish between no more than 7 plus minus 2 classes.
\end{abstract}

\section{Keywords}

Fuzzy logic, Non-associative operations

\section{Introduction}

In many application areas, there are tasks which take a lot of expert's time; example: interpreting the satellite photos. It is desirable to automate these time-consuming tasks.

One of the main obstacles to automating expert activity is the fact that experts often cannot express their activity in precise terms, they use vague (fuzzy) terms from natural language to describe it. For example, in satellite photo interpretation, an expert may follow a rule like "if an object is very small, it is probably a speckle unless a similar object appears on different photos of the same area"; here, "very small" and "similar" are examples of fuzzy terms from natural language.

To describe such fuzzy words, L. Zadeh proposed to use a special generalization of 2valued logic called fuzzy logic, in which a statement, in addition to being absolutely true and absolutely false, can also take additional truth values corresponding to uncertainty. How is fuzzy logic usually formalized ([4], [8])? There are many seemingly reasonable requirements that a logic should satisfy: e.g., since $A \& B$ and $B \& A$ are the same, the corresponding andoperation should be commutative. Similarly, since $A \& A$ means the same as $A$, we should expect that the and-operation should also satisfy this property, etc. It turns out to be impossible to satisfy all these seemingly natural requirements, so usually, some requirements are picked as absolutely true (like commutativity or associativity), and others are ignored if they contradict to the picked ones.

This idea leads to a neat mathematical theory, but the analysis of real-life expert reasoning shows that all the requirements are only approximately satisfied. Therefore, to achieve a more adequate representation of expert reasoning, instead of fixing some requirements as absolute and ignoring the others, we should require all of these requirements to be satisfied to some extent. In this paper, we show the preliminary results of analyzing such operations. 
In particular, we show that non-associative operations explain the empirical $7 \pm 2$ law in psychology according to which a person can normally distinguish between no more than 7 plus minus 2 classes.

\section{First Approach}

If we know the degrees of certainty (subjective probabilities) $p\left(S_{1}\right)$ and $p\left(S_{2}\right)$ in two statements $S_{1}$ and $S_{2}$, then possible values of $p\left(S_{1} \& S_{2}\right)$ form an interval

$$
p=\left[\max \left(p_{1}+p_{2}-1,0\right), \min \left(p_{1}, p_{2}\right)\right] .
$$

As a numerical estimate, it is natural to use a midpoint of this interval:

$$
\begin{gathered}
p_{1} \& p_{2} \stackrel{\text { def }}{=} \\
\frac{1}{2} \cdot \max \left(p_{1}+p_{2}-1,0\right)+\frac{1}{2} \cdot \min \left(p_{1}, p_{2}\right)
\end{gathered}
$$

Similar, for the "or"-operation, we can take the midpoint of the corresponding interval $\left[\max \left(p_{1}, p_{2}\right), \min \left(p_{1}+p_{2}, 1\right)\right]$ :

$$
\begin{gathered}
p_{1} \vee p_{2} \stackrel{\text { def }}{=} \\
\frac{1}{2} \cdot \max \left(p_{1}, p_{2}\right)+\frac{1}{2} \cdot \min \left(p_{1}+p_{2}, 1\right) .
\end{gathered}
$$

There is a problem with these operations. Indeed, any "and" operation $p_{1} \& p_{2}$ enables us to produce an estimate for $P\left(S_{1} \& S_{2}\right)$ provided that we know estimates $p_{1}$ for $p\left(S_{1}\right)$ and $p_{2}$ for $p\left(S_{2}\right)$. If we are interested in estimating the degree of belief in a conjunction of three statements $S_{1} \& S_{2} \& S_{3}$, then we can use the same operation twice:

- first, we apply the "and" operation to $p_{1}$ and $p_{2}$ and get an estimate $p_{1} \& p_{2}$ for the probability of $S_{1} \& S_{2}$;

- then, we apply the "and" operation to this estimate $p_{1} \& p_{2}$ and $p_{3}$, and get an estimate $\left(p_{1} \& p_{2}\right) \& p_{3}$ for the probability of $\left(S_{1} \& S_{2}\right) \& S_{3}$

Alternatively, we can get start by combining $S_{2}$ and $S_{3}$, and get an estimate $p_{1} \&\left(p_{2} \& p_{3}\right)$ for the same probability $p\left(S_{1} \& S_{2} \& S_{3}\right)$. Intuitively, we would expect these two estimates to coincide: $\left(p_{1} \& p_{2}\right) \& p_{3}=p_{1} \&\left(p_{2} \& p_{3}\right)$, i.e., in algebraic terms, we expect the operation \& to be associative. Unfortunately, midpoint operations are not associative: e.g.,

$$
(0.4 \& 0.6) \& 0.8=0.2 \& 0.8=0.1,
$$

while

$$
0.4 \&(0.6 \& 0.8)=0.4 \& 0.5=0.2 \neq 0.1 \text {. }
$$

By itself, a small non-associativity may not be so bad:

- associativity comes from the requirement that our reasoning be rational, while

- it is well known that our actual handling of uncertainty is not exactly following rationality requirements; see, e.g., [9].

So, it is desirable to find out how nonassociative can these operations be.

To be more precise, we know that the midpoint operations are non-associative, i.e., that sometimes, $(a \& b) \& c \neq a \&(b \& c)$. We want to know how big can the difference $(a \& b) \& c-a \&(b \& c)$ can be.

\section{Theorem 1 [2] .}

$$
\max _{a, b, c}|(a \& b) \& c-a \&(b \& c)|=\frac{1}{9} .
$$

\section{Theorem 2 [2] .}

$$
\max _{a, b, c}|(a \vee b) \vee c-a \vee(b \vee c)|=\frac{1}{9}
$$

Human experts do not use all the numbers from the interval $[0,1]$ to describe their possible degrees of belief; they use a few words like "very probable", "mildly probable", etc. Each of words is a "granule" covering the entire sub-interval of values. Since the largest possible non-associativity degree $|(a \& b) \& c-a \&(b \& c)|$ is equal to $1 / 9$, this non-associativity is negligible if the corresponding realistic "granular" degree of belief have granules of width $\geq 1 / 9$. One can fit no more than 9 granules of such width in the interval $[0,1]$. This may explain why humans are most comfortable with $\leq 9$ items to choose from - the famous "7 plus minus 2" law; see, e.g., $[5,6]$.

This general psychological law has also been confirmed in our specific area of formalizing expert knowledge: namely, in [1], it was shown that this law explains why in intelligent control, experts normally use $\leq 9$ different degrees (such as "small", "medium", etc.) to describe the value of each characteristic. 
Instead of selecting a midpoint, we can make a more general selection of a value in the interval $\mathbf{p}$. By a choice function, we mean a function $s$ that maps every interval $\mathbf{u}=$ $\left[u^{-}, u^{+}\right]$into a point $s(\mathbf{u}) \in \mathbf{u}$ so that for every $c$ and $\lambda>0$ :

- $s\left(\left[u^{-}+c, u^{+}+c\right]\right)=s\left(\left[u^{-}, u^{+}\right]\right)+c$ (shift-invariance);

- $s\left(\left[\lambda \cdot u^{-}, \lambda \cdot u^{+}\right]\right)=\lambda \cdot s\left(\left[u^{-}, u^{+}\right]\right)$ (unit-invariance).

Proposition 1 [7] . Every choice function has the form $s\left(\left[u^{-}, u^{+}\right]\right)=\alpha \cdot u^{-}+(1-\alpha) \cdot u^{+}$ for some $\alpha \in[0,1]$.

The combination $p=\alpha \cdot p^{-}+(1-\alpha) \cdot p^{+}$ (first proposed by Hurwicz [3]) has been successfully used in areas ranging from submarine detection to petroleum engineering [7]; in [11], this approach is applied to second-order probabilities.

With this approach, we get the following formulas which generalize the above definitions:

$$
\begin{gathered}
p_{1} \& p_{2} \stackrel{\text { def }}{=} \alpha \cdot \max \left(p_{1}+p_{2}-1,0\right)+ \\
(1-\alpha) \cdot \min \left(p_{1}, p_{2}\right) ; \\
p_{1} \vee p_{2} \stackrel{\text { def }}{=} \alpha \cdot \max \left(p_{1}, p_{2}\right)+ \\
(1-\alpha) \cdot \min \left(p_{1}+p_{2}, 1\right) .
\end{gathered}
$$

Theorem 3 [2] .

$$
\begin{gathered}
\max _{a, b, c}|(a \& b) \& c-a \&(b \& c)|= \\
\frac{\alpha \cdot(1-\alpha)}{2+\alpha \cdot(1-\alpha)} ; \\
\max _{a, b, c}|(a \vee b) \vee c-a \vee(b \vee c)|= \\
\frac{\alpha \cdot(1-\alpha)}{2+\alpha \cdot(1-\alpha)} .
\end{gathered}
$$

Comment. This non-associativity degree is the smallest $(=0)$ when $\alpha=0$ or $\alpha=1$, and the largest $(=1 / 9)$ for midpoint operations $(\alpha=$ $0.5)$.

In our proof, it was useful to first show that the new operations have some properties of associativity: namely, it turns out that for every $\alpha$, both operations are semi-associative in the sense that $a \leq b \leq c$ implies that $a \&(b \& c) \geq$ $b \&(a \& c) \geq c \&(a \& b)$.

\section{Second Approach}

A t-norm $a \& b$ describes the degree to which two conditions $A$ and $B$ are both satisfied if we know that the first condition $A$ is satisfied with a degree $a$, and the second condition $B$ is satisfied with a degree $b$.

In effect, t-norms describe the situations when both conditions are absolutely necessary, so that if one of the conditions is not satisfied, we completely reject the corresponding alternative. There are many such situations, but there are also many other situations, in which, although we say that we want the first condition to be satisfied and the second condition to be satisfied, etc., but if one of these conditions is not satisfied, we may still consider the corresponding alternative.

For example, a computer science department may be looking for a person who is a brilliant researcher and a very good lecturer and is knowledgeable in all the areas of computer science, i.e., in data structures and in operating systems and in software engineering etc. Ideally, all these conditions should be met. However, if a brilliant researcher with a reputation of a good lecturer applies for a position, then, even if he does not know anything about operating systems, a department would most probably not definitely reject him.

In short, in many real-life situations, even if one of the conditions $A, B$ is not satisfied at all, e.g., if $a=0$, we may still have some non-zero degree of belief in the conjunction $A \& B$ - in direct contrast to the fact that for a t-norm, in this case, $0 \& b=0$. This difference between the formal notion of a t-norm and the human use of "and" was noticed several decades ago, in the experiments of H.-J. Zimmermann and P. Zysno described in [12]. To get a more adequate description of human "and"-operations, the authors of [12] propose to use, instead of tnorm, a combination (e.g., linear combination) of a t-norm and a t-conorm, e.g., to use a combination

$$
\begin{gathered}
p_{1} \& p_{2} \stackrel{\text { def }}{=} \\
\alpha \cdot \min \left(p_{1}, p_{2}\right)+(1-\alpha) \cdot \max \left(p_{1}, p_{2}\right) . \quad(5)
\end{gathered}
$$

Such a combination is also not associative. How non-associative can it be? To answer this question, we prove that it is semi-associative:

Proposition 2. If $a \geq b \geq c$, then

$$
a \&(b \& c) \geq b \&(a \& c) \geq c \&(a \& b) .
$$




\section{Theorem 4.}

$$
\max _{a, b, c}|(a \& b) \& c-a \&(b \& c)|=\alpha \cdot(1-\alpha) .
$$

Proof of Proposition 2 and Theorem 4. Let $a \geq b \geq c$.

$1^{\circ}$. Let us first prove that

$$
a \&(b \& c) \geq b \&(a \& c) .
$$

Indeed, in this case, $b \& c=(1-\alpha) \cdot b+\alpha \cdot c$. Since $b \leq a$ and $c \leq a$, we can conclude that $b \& c \leq a$. Therefore,

$$
\begin{aligned}
& a \&(b \& c)=(1-\alpha) \cdot a+\alpha \cdot(b \& c)= \\
& (1-\alpha) \cdot a+\alpha \cdot(1-\alpha) \cdot b+\alpha^{2} \cdot c .
\end{aligned}
$$

Similarly, $a \& c=(1-\alpha) \cdot a+\alpha \cdot c$. The expression for $b \&(a \& c)$ depends on whether $b \geq(a \& c)$ or not. Correspondingly, let us consider both cases.

$1 \cdot 1^{\circ}$. Let us first consider the case when

$$
b \geq(a \& c) .
$$

In this case,

$$
b \geq(1-\alpha) \cdot a+\alpha \cdot c
$$

hence

$$
\begin{aligned}
& b \&(a \& c)=\alpha \cdot(a \& c)+(1-\alpha) \cdot b= \\
& \alpha \cdot(1-\alpha) \cdot a+(1-\alpha) \cdot b+\alpha^{2} \cdot c .
\end{aligned}
$$

The difference between the expressions (6) and (8) is equal to $(1-\alpha)^{2} \cdot(a-b)$, so this difference is non-negative. For this case, the desired inequality is proven.

$1.2^{\circ}$. Let us now consider the case when

$$
b<(a \& c) \text {. }
$$

In this case,

$$
b<(1-\alpha) \cdot a+\alpha \cdot c,
$$

hence

$$
\begin{aligned}
& b \&(a \& c)=(1-\alpha) \cdot(a \& c)+\alpha \cdot b= \\
& (1-\alpha)^{2} \cdot a+\alpha \cdot b+\alpha \cdot(1-\alpha) \cdot c .
\end{aligned}
$$

The difference between the expressions (6) and (10) is equal to:

$$
\begin{gathered}
\alpha \cdot(1-\alpha) \cdot a-\alpha^{2} \cdot b+\alpha \cdot(2 \alpha-1) \cdot c= \\
\alpha \cdot \sigma,
\end{gathered}
$$

where by $\sigma$, we denoted the expression

$$
(1-\alpha) \cdot a-\alpha \cdot b+(2 \alpha-1) \cdot c .
$$

Due to (9), we have

$$
\alpha \cdot b<\alpha \cdot(1-\alpha) \cdot a+\alpha^{2} \cdot c,
$$

hence

$\sigma>(1-\alpha) \cdot a-\alpha \cdot(1-\alpha) \cdot a-\alpha^{2} \cdot c+(2 \alpha-1) \cdot c=$

$(1-\alpha)^{2} \cdot a-(1-\alpha)^{2} \cdot c=(1-\alpha)^{2} \cdot(a-c)$.

Since $a \geq c$, we conclude that $\sigma \geq 0$, hence the difference between (6) and (10) is also nonnegative. So, for this second case, the desired inequality is also proven.

$2^{\circ}$. Let us now prove that

$$
b \&(a \& c) \geq c \&(a \& b) .
$$

Since $a \geq b$, we have $a \& b=(1-\alpha) \cdot a+\alpha \cdot b$. From $a \geq c$ and $b \geq c$, we conclude that

$$
a \& b=(1-\alpha) \cdot a+\alpha \cdot b \geq c .
$$

Thus,

$$
\begin{aligned}
& c \&(a \& b)=(1-\alpha) \cdot(a \& b)+\alpha \cdot c= \\
& (1-\alpha)^{2} \cdot a+\alpha \cdot(1-\alpha) \cdot b+\alpha \cdot c .
\end{aligned}
$$

To prove the desired inequality, we consider the same two cases as in Part 1 of this proof.

$2.1^{\circ}$. Let us first consider the case when

$$
b \geq(a \& c) .
$$

In this case, $b \&(a \& c)$ is described by the expression (8). The difference between the expressions (8) and (11) is equal to

$(1-\alpha) \cdot(2 \alpha-1) \cdot a+(1-\alpha)^{2} \cdot b-\alpha \cdot(1-\alpha) \cdot c=$

$$
(1-\alpha) \cdot \sigma
$$

where by $\sigma$, we denoted the expression:

$$
(2 \alpha-1) \cdot a+(1-\alpha) \cdot b-\alpha \cdot c .
$$

Due to (7), we have

$$
(1-\alpha) \cdot b \geq(1-\alpha)^{2} \cdot a+\alpha \cdot(1-\alpha) \cdot c,
$$

hence

$$
\begin{gathered}
\sigma \geq(2 \alpha-1) \cdot a+(1-\alpha)^{2} \cdot a+\alpha \cdot(1-\alpha) \cdot c-\alpha \cdot c= \\
\alpha^{2} \cdot(a-c) .
\end{gathered}
$$

Since $a \geq c$, we conclude that $\sigma \geq 0$, hence the difference between (8) and (11) is also nonnegative. So, for this case, the desired inequality is proven. 
$2.2^{\circ}$. Let us first consider the case when

$$
b<(a \& c) \text {. }
$$

In this case, $b \&(a \& c)$ is described by the expression (10). The difference between the expressions (10) and (11) is equal to $\alpha^{2} \cdot(b-c)$. Since $b \geq c$, this difference is non-negative, hence the desired inequality holds in this case too.

This completes the proof of Proposition 2.

$3^{\circ}$. Let us now prove Theorem 5 .

Since every three real numbers can be sorted in the order $a \geq b \geq c$, to prove Theorem 5, it is sufficient to consider all possible differences between the terms $a \&(b \& c), b \&(a \& c)$, and $c \&(a \& b)$ that correspond to $a \geq b \geq c$.

Due to Proposition 2, the largest possible difference $d$ between these three terms is the difference between the expressions $a \&(b \& c)$ and $c \&(a \& b)$. The first expression is described by the formula (6), the second by the formula (11), thus, the difference between these expressions is equal to the difference between these formulas, i.e., to:

$$
\begin{gathered}
d=\alpha \cdot(1-\alpha) \cdot a-\alpha \cdot(1-\alpha) \cdot c= \\
\alpha \cdot(1-\alpha) \cdot(a-c) .
\end{gathered}
$$

Since $a \geq c$, the difference $a-c$ can take values between 0 and 1 , the largest value 1 attained when $a=1$ and $c=0$. Thus, $d \leq \alpha \cdot(1-\alpha)$, and $d=\alpha \cdot(1-\alpha)$ when $a=1$ and $c=0$. Hence, the desired maximum of the difference $d$ is indeed equal to $\alpha \cdot(1-\alpha)$. The theorem is proven.

\section{Third Approach}

In the above text, we only talked about "and" and "or" operations. What about more complex logical operations? If we fix "and", "or", and "not" operations, then we can, in principle, knowing the degree of belief in the basic statements, determine the degree of belief in their logical combination $Q$. To do that, we represent the given formula $Q$ as a combination of $\&, \vee$, and $\neg$, and then consequently use our chosen operations with degrees of belief instead of these logical symbols.

There is a problem with this approach: Every expression can be described in several different ways in terms of the basic logical operations $\&, \vee$, and $\neg$. For example, $A \rightarrow B$ can be represented as $B \vee \neg A,(A \& B) \vee \neg A$ etc. These expressions are equivalent in normal
Boolean (2-valued) logic, but if we use these expression to compute degrees of belief, we sometimes end up with different results. E.g., in the above case, if $d(A)=0.6$ and $d(B)=0.7$, and we use min, $\max$, and $x \rightarrow 1-x$ for $\&, \vee$, and $\neg$, then the first expression leads to $\max (d(B), 1-d(A))=0.7$, while the second leads to

$$
\begin{gathered}
\max (\min (d(A), d(B)), 1-d(A))= \\
\max (0.6,0.4)=0.6 .
\end{gathered}
$$

So, for as given expression $F$, instead of a single value of $d(F)$, we end up with different possible values $p_{F}(a, \ldots, b)$ of $d(F)$. It is therefore desirable to describe the interval formed by the smallest and the largest possible values of $d(F)$ for all $F$ that correspond to a given formula. This idea was first described by Türkşen in [10]. It turns out that if we use min and max, then the smallest and the largest values can be explicitly described.

By a propositional formula in a DNF (disjunctive normal form), we mean a formula of the type $C_{1} \vee \ldots \vee C_{m}$, where each $C_{j}$ is of the type $x_{1} \& \ldots \& x_{p}$, and $x_{i}$ are either the basic statements or their negations. We say that we have a complete $D N F$ if each $C_{j}$ contains all variables from the formula.

By a propositional formula in a CNF (conjunctive normal form), we mean a formula of the type $D_{1} \& \ldots \& D_{m}$, where each $D_{j}$ is of the type $x_{1} \vee \ldots \vee x_{p}$, and $x_{i}$ are either the basic statements or their negations. We say that we have a complete $C N F$ if each $D_{j}$ contains all variables from the formula.

Every propositional formula can be transformed into a unique complete $\mathrm{CNF}$ or into a uniquely defined complete DNF form. These unique formulas will be denoted by $\operatorname{CNF}(F)$ and $\operatorname{DNF}(F)$. For example, $A \rightarrow B$ can be transformed into a complete $\mathrm{CNF}$ form $\neg A \vee B$, or into a complete DNF form $(A \& B) \vee(\neg A \& B) \vee(\neg A \& \neg B)$.

Proposition 3 [13] . Let \& $=\min , \mathrm{V}=\max$, and $\neg(x)=1-x$. Then, for every propositional formula $F(A, \ldots, B)$, and for all values $a, \ldots, b$,

$$
\begin{gathered}
p_{\mathrm{DNF}(\boldsymbol{F})}(a, \ldots, b) \leq p_{F}(a, \ldots, b) \leq \\
p_{\mathrm{CNF}(\boldsymbol{F})}(a, \ldots, b) .
\end{gathered}
$$

So, for every formula $F$, we can take 


$$
\left[p_{\mathrm{DNF}(F)}(a, \ldots, b), p_{\mathrm{CNF}(F)}(a, \ldots, b)\right]
$$

as the desired interval. In particular, for the $F=A \& B$, we get the interval $\left[p^{-}, p^{+}\right]$, where: $p^{-}=\min (a, b)$, and $p^{+}$is equal to

$\min (\max (1-a, b), \max (a, 1-b), \max (a, b))$, and for $F=A \vee B$, we get the interval $\left[p^{-}, p^{+}\right]$, where $p^{-}$is equal to

$\max (\min (1-a, b), \min (a, 1-b), \min (a, b))$, and $p^{+}=\max (a, b)$. For these intervals, Hurwicz criterion leads to the following operations:

$$
a \& b \stackrel{\text { def }}{=} \alpha \cdot \min (a, b)+(1-\alpha) \cdot(a \otimes b),
$$

where

$$
a \otimes b=\stackrel{\text { def }}{=}
$$

$\min (\max (1-a, b), \max (a, 1-b), \max (a, b))$, and

$$
a \vee b \stackrel{\text { def }}{=} \alpha \cdot(a \oplus b)+(a-\alpha) \cdot \max (a, b),
$$

where

$$
\begin{aligned}
& a \oplus b \stackrel{\text { def }}{=} \\
& \max (\min (1-a, b), \min (a, 1-b), \min (a, b)) .
\end{aligned}
$$

\section{Theorem 5.}

$$
\begin{aligned}
& \max _{a, b, c}|(a \& b) \& c-a \&(b \& c)|=\frac{\alpha \cdot(1-\alpha)}{2} ; \\
& \max _{a, b, c}|(a \vee b) \vee c-a \vee(b \vee c)|=\frac{\alpha \cdot(1-\alpha)}{2} .
\end{aligned}
$$

\section{Acknowledgments}

This work was supported in part by NASA grants NCC5-209 and NCC 2-1232, by the Air Force Office of Scientific Research grant number F49620-00-1-0365, by Grant No. W-00016 from the U.S.-Czech Science and Technology Joint Fund, and by Grant NSF 9710940 Mexico/Conacyt.

\section{References}

[1] L. Godo, R. Lopez de Mantaras, C. Sierra, and A. Verdaguer, MILORD: The Architecture and management of Linguistically expressed Uncertainty, International Journal of Intelligent Systems, 1989, Vol. 4, pp. 471-501.
[2] I. R. Goodman, R. A. Trejo, V. Kreinovich, J. Martinez, and R. Gonzalez, An even more realistic (non-associative) interval logic and its relation to psychology of human reasoning, Proceedings of the Joint 9th World Congress of the International Fuzzy Systems Association and 20th International Conference of the North American Fuzzy Information Processing Society IFSA/NAFIPS 2001, Vancouver, Canada, July 25-28, 2001 (to appear).

[3] L. Hurwicz, A criterion for decisionmaking under uncertainty, Technical Report 355, Cowles Commission, 1952.

[4] G. Klir and B. Yuan, Fuzzy Sets and Fuzzy Logic: Theory and Applications, Prentice Hall, Upper Saddle River, NJ, 1995.

[5] G. A. Miller, The magical number seven plus or minus two: some limits on our capacity for processing information, Psychological Review, 1956, Vol. 63, pp. 81-97.

[6] P. M. Milner, Physiological psychology, Holt, NY, 1970.

[7] H. T. Nguyen and V. Kreinovich, Nested Intervals and Sets: Concepts, Relations to Fuzzy Sets, and Applications, In: R. B. Kearfott and V. Kreinovich (eds.), Applications of Interval Computations, Kluwer, Dordrecht, 1996, pp. 245-290.

[8] H. T. Nguyen and E. A. Walker, First Course in Fuzzy Logic, CRC Press, Boca Raton, FL, 1999.

[9] P. Suppes, D. M. Krantz, R. D. Luce, and A. Tversky, Foundations of measurement, Vol. I-III, Academic Press, San Diego, CA, 1989.

[10] I. B. Türkşen, Interval valued fuzzy sets based on normal forms, Fuzzy Sets and Systems, 1986, Vol. 20, pp. 191-210.

[11] T. Whalen, Interval probabilities induced by decision problems, In: R. R. Yager, J. Kacprzyk, and M. Pedrizzi (Eds.), Advances in the Dempster-Shafer Theory of Evidence, Wiley, N.Y., 1994, pp. 353-374.

[12] H. H. Zimmerman and P. Zysno, Latent connectives in human decision making, Fuzzy Sets and Systems, 1980, Vol. 4, pp. 37-51.

[13] Q. Zuo, I. B. Türkşen, H. T. Nguyen, and V. Kreinovich, In expert systems, even if we fix AND/OR operations, a natural answer to a composite query is the interval of possible degrees of belief, Reliable Computing, 1995, Supplement (Extended Abstracts of APIC'95: International Workshop on Applications of Interval Computations, El Paso, TX, Febr. 23-25, 1995), pp. 236-240. 